This is a postprint version of the following published document:

D. S. Montero, J. C. Torres, J. Z. Viñuelas, J.L. P. Castellanos, C. Vázquez."Effects of elongation on polymer optical fiber power losses for sensing purposes". Proc. SPIE 9157, 23rd International Conference on Optical Fibre Sensors, 91579W (June 2, 2014). Available in doi:10.1117/12.2059276

(C) 2009 Society of Photo Optical Instrumentation Engineers. One print or electronic copy may be made for personal use only. Systematic electronic or print reproduction and distribution, duplication of any material in this paper for a fee or for commercial purposes, or modification of the content of the paper are prohibited. 


\title{
Effects of elongation on polymer optical fiber power losses for sensing purposes
}

\author{
D. S. Montero*a , J. C. Torres ${ }^{\mathrm{a}}$, J. Zahr-Viñuelas ${ }^{\mathrm{b}}$, J. L. Pérez-Castellanos ${ }^{\mathrm{b}}$, C. Vázquez ${ }^{\mathrm{a}}$ \\ ${ }^{a}$ Electronics Technology Dpt., Universidad Carlos III de Madrid, Avda. de la Universidad 30, 28911, \\ Leganés (Madrid), Spain; ${ }^{b}$ Continuum Mechanics and Structural Analysis Dpt., Universidad Carlos \\ III de Madrid, Avda. de la Universidad 30, 28911, Leganés (Madrid), Spain
}

\begin{abstract}
In this work, we explore the variation of power losses of PMMA-core plastic optical fiber through tensile tests. Experimental results of received optical power during elastic and plastic deformation process demonstrate the feasibility of POFs as simple intensity-based fiber-optic sensors for Structural Health Monitoring applications beyond complex schemes. A theoretical approach of the POF behavior versus strain is also provided.
\end{abstract}

Keywords: Plastic Optical Fiber (POF), POF mechanical behavior, Structural Health Monitoring (SHM)

\section{INTRODUCTION}

A novel market niche constitutes structural health monitoring (SHM) purposes. In this latter scenario, strain measurement is of most interest for structural analysis, such as cracking detection or fatigue analysis. Compared to glass optical fibers, POFs are cheaper and easier to align thus driving cost-effective sensing approaches. Related to standard PMMA POF fibers, they can be strained to more than $40 \%$ due to their elasto-plastic properties while fully maintaining their light guiding properties. This makes then well-suited for SHM applications when compared, for instance, to current FBG (Fiber Bragg Grating)-based solutions. These are mostly based on glass optical fiber which provides limited measurement ranges due to their low break-down strain of only percentage units of strain (typically $1 \%$ ).

Focusing on POF-based SHM solutions, different techniques employing POF for strain measurements have been reported in literature with great success. Since a Bragg grating can be written in a single mode POF ${ }^{1}$, the POF has become an attractive optical device in sensor systems for the aforementioned applications. Nevertheless, the drawback relies on the fact that typically an Optical Spectrum Analyzers (OSA) needs to be used to monitor the wavelength shift versus strain by its principle of operation. This fact usually leads to a dramatic increase of total cost of the solution employed $^{2}$. Additionally, such POF-based FBGs also suffer from higher losses and temperature dependence, becoming a constraint in applications in which temperature is seen as an external undesirable factor. In other cases, the SHM monitoring technique employs singlemode POFs extended to POF-based interferometric schemes allowing high accuracy for such measurements but increasing the complexity of the total system ${ }^{3}$. Finally, other authors have shown that distributed strain sensing with POF by evaluating the distributed Rayleigh backscatter signal using Optical Time Domain Reflectometry (OTDR) is possible ${ }^{4}$.

The principle of the intensity-based measurement implemented for SHM applications have been reported in literature by different authors 5,6 , where the POF needed to be bent. In that cases, the fewer propagating modes experience total internal reflection at the location of the bend, and they are therefore radiated decreasing the total optical power received. A nearly constant normalized optical power ratio slope (i.e. sensitivity) in POF specimens subjected to different bending radii has been reported ${ }^{7}$. In other approaches the POF is simply embedded with no bending to monitor the crack evolution in composite laminates ${ }^{8}$. However, little effort has been made on determining the characteristics of such POF sensors versus the applied strain rate.

In this work, POF specimens with no need for fiber bending when embedding or gluing in structural elements being subjected to deformational states are investigated at different strain rates. The paper is organized as follows: first, the theoretical background is briefly described in Section 2; next, the variations in POF power loss for various strain rates are reported and analyzed in Section 3; finally, conclusions are provided in Section 4.

* dsmontero@ing.uc3m.es; phone +34916248865; fax +34916249430 


\section{THEORETICAL BACKGROUND}

The mechanical deformation of a fiber consists of a simultaneous axial elongation and diameter decrease. Both effects are altered due to stress/strain and may change the refractive index of the optical fiber thus affecting the optical performance. Theoretically, this deformation can cause anisotropic and inhomogeneous distribution of the refractive index, a process which is called photo-elastic effect. It has been estimated a refractive index change caused by stress in the order of $\pm 0.01^{9}$. On the other hand, when the light propagates from the unstressed to the stressed area if the refractive index contrast between the core and cladding regions decreases, the field confinement becomes weak thus increasing the power loss. This phenomenon is referred to as radiation confinement loss ${ }^{10}$. Particularly, in POFs, previous works have demonstrated that the PMMA (core material) refractive index decreases with strain whereas fluorinated polymers (cladding material) increase their refractive index directly proportional to the applied $\operatorname{strain}^{11}$.

The variation of the refractive index due to stress can be expressed, from the common nomenclature standard and notation, as a function of both the stress-optic coefficients, $c_{i}$ and the applied stress, $\sigma_{i j}$, thus yielding $\Delta n_{i j}=F\left(c_{i}, \sigma_{i j}\right)$. Moreover, $\Delta n_{i j}$ can also be expressed as a function of strain, $\varepsilon$, by $\sigma_{i j}=E \cdot \varepsilon_{i j}$ in which $E$ corresponds to the Young's modulus. On the other hand, this refractive index variation can be alternatively expressed as a function of the axial strain energy density by means of $U_{a}=\int_{0}^{\varepsilon} \sigma d \varepsilon$. The overall axial strain energy, $\bar{U}_{A}$ is given by:

$$
\bar{U}_{A}=\int_{V} U_{a} d V=\int_{L} U_{a} d z \text { with } U_{a}=\frac{1}{2} E A\left(\frac{d \delta}{d z}\right)^{2}
$$

where $V$ is the total volume of the POF specimen, $A$ is the fiber cross-sectional area and $\delta$ is the change in length due to deformation process, respectively. It has been assumed that fiber diameter variations due to the applied load have a negligible impact on the optical fiber power loss.

\section{EXPERIMENTAL RESULTS}

A standard $980 \mu \mathrm{m}$ core-diameter step-index POF fiber was employed during tensile tests. This fiber type has a cladding with $0.01 \mathrm{~mm}$ of thickness composed of a fluorinated polymer (typically fluoroalkylmethacrylate) thus having a lower refractive index than that of the fiber core and assuring light propagation condition through the fiber.

(a)

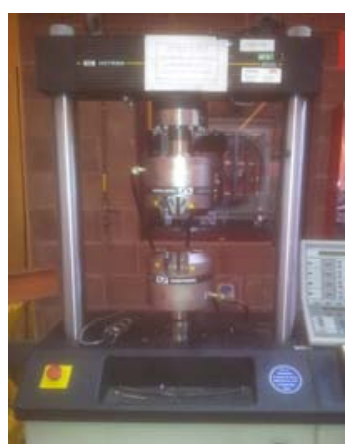

Figure 1. (a) Picture of the testing machine for strain measurements, (b)

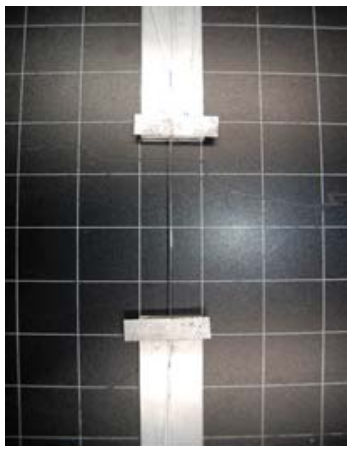

(b) POF specimen.

Quasi-static tests controlling the displacement of the bare POF fiber specimens were performed. Fig. 1a shows a picture of the testing machine (INSTRON model 8516/8802) used for the deformation trials, with a maximum load capacity of 100/250kN. Fig. 1b depicts a POF specimen especially designed to prevent undesirable fiber skids during the data acquisition that could distort the measurements. 
From each test the true stress-true strain $(\sigma-\varepsilon)$ relation and mechanical parameters such as Young's modulus, E, yield strength, $\sigma_{\mathrm{y}}$, and the tensile strength, $\mathrm{S}_{\mathrm{m}}$ are obtained. Statistically negligible differences between the results of different test under the same conditions are observed. Figure 2 depicts an illustrative example of a stress-strain relation obtained in a test at strain rate $0.0025 \mathrm{~s}^{-1}$. The shape of the curve reveals the deformation process of the PMMA material under applied strain. Beyond the elastic limit, the first negative slope of the curve and further significant change (beyond $(\varepsilon \sim 0.1)$ is attributed to the reorientation of the PMMA molecular chains in the same axis of the applied load. The measured mechanical properties at different strain rates are depicted in Fig. 2(b). As a matter of fact, increasing the strain rate results in a slight increase of the Young's modulus, whereas the yield strength and the tensile strength increase significantly.

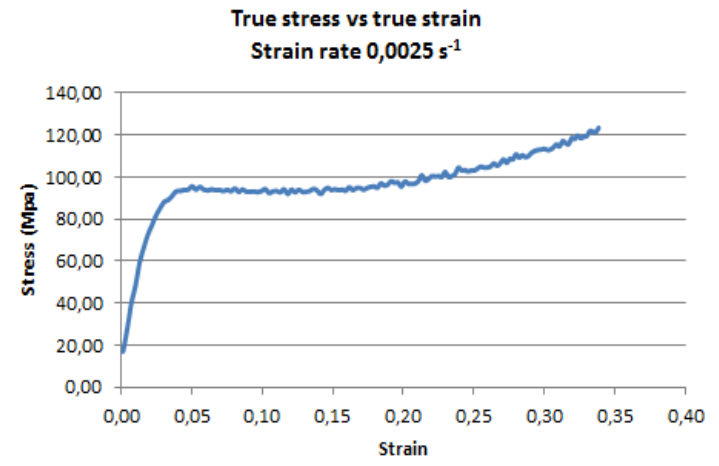

Figure 2. (a) POF stress-strain curve at a strain rate of $0.0025 \mathrm{~s}^{-1}$.

\begin{tabular}{|l|c|c|}
\hline \multicolumn{1}{|c|}{ Strain rate } & $\dot{\varepsilon}=0,0025 \mathrm{~s}^{-1}$ & $\dot{\varepsilon}=0,0068 \mathrm{~s}^{-1}$ \\
\hline Young's modulus, $\mathbf{M P a}$ & 3850 & 3930 \\
\hline Yield strength, $\mathbf{M P a}$ & $85-87$ & $105-108$ \\
\hline Tensile strength, $\mathbf{M P a}$ & 120 & 150 \\
\hline
\end{tabular}

(b) Measured mechanical properties of POF.

The scheme used to measure the POF power loss during the mechanical characterization consisted on a LED source operating at $635 \mathrm{~nm}$ and a receiver driver including both a photodiode and electronic amplifying/filtering stages. Experimental data is recorded by means of a digital oscilloscope for an off-line signal processing. To avoid undesirable variations in the measured power loss due to short-term optical source fluctuations or external perturbations that could distort the analysis of the experimental results, a self-referencing scheme is implemented. Another POF is deployed obtaining a reference signal by bypassing the sensing specimen, thus providing a self-referencing spatial separation technique.

(a)

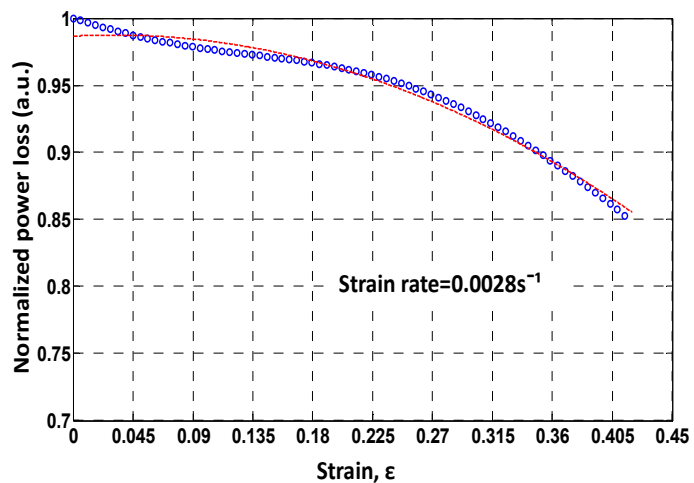

(b)

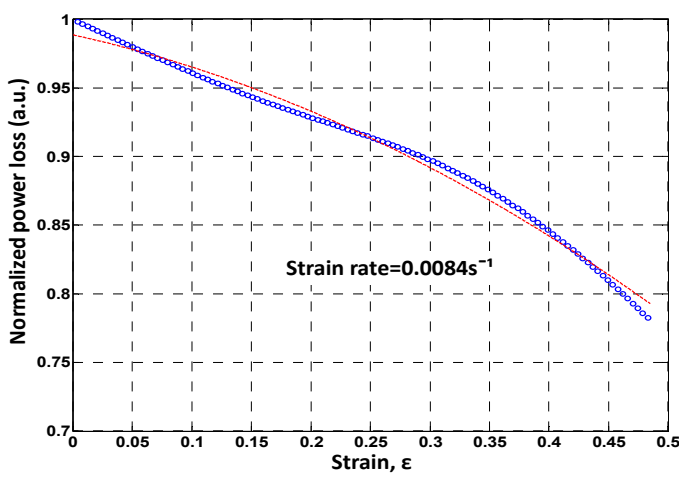

Figure 3. Normalized power loss versus applied strain for various strain rates: (a) $0.0028 \mathrm{~s}^{-1}$, (b) $0.0084 \mathrm{~s}^{-1}$.

To enhance the interaction between the power loss and the applied strain, we normalize the received optical power as $\mathrm{P}_{\text {out }} / \mathrm{P}_{\text {out }}^{\varepsilon=0}$, where the reference value is the measured power loss of the specimen when no strain is applied. Fig. 3 shows two illustrative cases of the effect on power ratio for POF specimens subjected to different strain rate tests. It is clearly noticed that the received optical power decreases as the elongation increases. Nevertheless, little influence of the power loss versus the applied strain rate is noticeable. The reduction in the measured power ratio is in the order of 15$20 \%$ for larger strain, i.e. before breaking. Upper POF strain limit before failure resulted in $\varepsilon=0.49$. Nevertheless, all the specimens tested showed a uniform behavior in which this limit was in a range from $\varepsilon=0.40-0.49$. Dashed lines in both 
figures represent the second-order polynomial curve fit of the measured normalized power loss. For our estimation, we have considered a quadratic dependence of the received optical power, given by Eq. (1). Upper bound of the relative error in all cases was $9 \%$, although error average of all trials was approximately $5 \%$.

\section{CONCLUSIONS}

We report experimental trials for bare PMMA-core POF specimens under axial strain with simultaneous measurements of the optical power detected at reception. From the results, it is shown that the received optical power decreases as the elongation increases, although little dependence with the applied strain rate is noticeable. This fact is explained because the refractive index contrast between the core and cladding regions decreases, when the fiber is subjected to a deformation process. Consequently the field confinement becomes weak thus increasing the power loss.

Moreover, this power loss versus the applied strain shows a quadratic dependence, directly related to the induced strain energy variation stored in the deformed POF specimen. Under the assumption of quadratic dependence, errors in the quadratic estimation are in the order of units of percentage. But, what it is most important, the reduction in the measured power ratio is in the order of $15-20 \%$ demonstrating that cost-effective POF-based intensity-based sensors may be suitable as part of Structural Health Monitoring (SHM) systems beyond complex schemes under specific conditions. No need for fiber extra bending, when embedding or gluing in structural elements being subjected to deformational states, is required. We are currently working in a detailed theoretical approach which will be reported elsewhere.

\section{ACKNOWLEDGEMENTS}

This work has been sponsored by the Spanish Ministry of Economía y Competividad under grant TEC2012-37983-C0302 .

\section{REFERENCES}

[1] Xiong, Z., Peng, G. D., Wu, B. and Chu P. L., "Highly tunable Bragg gratings in single-mode polymer optical fibers," IEEE Phot. Tech. Lett., 11(3), 352-354 (1999).

[2] Barrera, D., Muñoz, G. and Sales S., "Design, fabrication and characterization of a sensor for large strain measurement based on POFBGs," Reunión Nacional Española de Optoelectrónica (OPTOEL), 79-82 (2013).

[3] Kiesel, S., Peters, K., Abdi, O., Hassan, T. and Kowalsky, M., "Polymer optical fiber sensors for civil infrastructure systems," Proc. SPIE Sensors and Smart Structures Technologies for Civil, Mechanical and Aerospace Systems 6529, 65293B (2007).

[4] Liehr, S., Lenke, P., Krebber, K., Seeger, M., Thiele, E., Metschies, H., Gebreselasie, B., Münich J. C. and Stempniewski, L., "Distributed strain measurement with polymer optical fibers integrated into multifunctional geotextiles," Proc. SPIE Optical Sensors 7003, paper 700302 (2008).

[5] Kuang, K. S. C., Cantwell, W. J. and Thomas, C., "Crack detection and vertical deflection monitoring in concrete beams using plastic optical fiber sensors," Meas. Sci. Technol., 14, 205-216 (2003).

[6] Babchenko, A., Weinberger, Z., Itzkovich, N. and Maryles, J., "Plastic optical fibre with structural imperfections as a displacement sensor, “Meas. Sci. Technol., 17, 1157-1161 (2006).

[7] Chen, Y., Kuang, J., Chen, L., Chuang, H., "Effect of plastic strain energy strain density on polymer optical fiber power losses," Opt. Lett., 31, 879-881 (2006).

[8] Takeda, N. "Characterization of microscopic damage in composite laminates and real-time monitoring by embedded optical fiber sensors," International Journal of Fatigue, 24, 281-289 (2002).

[9] Huang, M., "Stress effects on the performance of optical waveguides," Int. J. Solids and Structures, 40, 16151632 (2003).

[10] Adams, M. J., [An Introduction to Optical Waveguide], John Wiley and Sons, Chichester (1981).

[11] Silva-López, M.A., Fender, W. N., MacPherson, W. N., Barton, J. S., Jones, J. D. C., Zhao, D., Webb, D. J., Zhang, L., Bennion, I., "Strain and termperature sensitivity of a singlemode polymer optical fibre," Opt. Lett., 30, 3129-3131 (2005). 